\title{
Aligned, Isotropic and Patterned Carbon Nanotube Substrates that Control the Growth and Alignment of Chinese Hamster \\ Ovary Cells
}

Che Azurahanim Che Abdullah ${ }^{1, \dagger}$, Piyapong Asanithi ${ }^{1,2, \dagger}$, Eric W Brunner ${ }^{1}$, Izabela Jurewicz ${ }^{1}$, Chiara Bo ${ }^{1,3}$, Chihye Lewis Azad ${ }^{4}$, Raquel Ovalle-Robles ${ }^{4}$, Shaoli Fang ${ }^{4}$, Marcio D Lima ${ }^{4}$, Xavier Lepro ${ }^{4}$, Steve Collins ${ }^{4}$, Ray H Baughman ${ }^{4}$, Richard P Sear ${ }^{1, *}$, and Alan B Dalton ${ }^{1}$

${ }^{1}$ Department of Physics and Surrey Materials Institute, University of Surrey, Guildford, Surrey GU2 7XH, United Kingdom

${ }^{2}$ Department of Physics, King Mongkut's University of Technology Thonburi, Bangkok 10140, Thailand

${ }^{3}$ Dipartimento di Ingegneria Biofisica ed Elettronica (DIBE), Università di Genova, via Opera Pia 11A, 16145 Genova, Italy

${ }^{4}$ The Alan G MacDiarmid NanoTech Institute, The University of Texas at Dallas, Richardson, TX 75080-3021, USA

${ }^{\dagger}$ These authors contributed equally to this work.

*r.sear@surrey.ac.uk 


\begin{abstract}
Here we culture Chinese Hamster Ovary cells on isotropic, aligned and patterned substrates based on multiwall carbon nanotubes. The nanotubes provide the substrate with nanoscale topography. The cells adhere to and grow on all substrates, and on the aligned substrate, the cells align strongly with the axis of the bundles of the multiwall nanotubes. This control over cell alignment is required for tissue engineering; almost all tissues consist of oriented cells. The aligned substrates are made using straightforward physical chemistry techniques from forests of multiwall nanotubes, no lithography is required to make inexpensive largescale substrates with highly aligned nanoscale grooves. Interestingly, although the cells strongly align with the nanoscale grooves, only a few also elongate along this axis: alignment of the cells does not require a pronounced change in morphology of the cell. We also pattern the nanotube bundles over lengthscales comparable to the cell size and show that the cells follow this pattern.
\end{abstract}




\section{Introduction}

Cells interact with, and respond to, the substrate they are growing on. Their adhesion and growth depends on substrate properties such as its stiffness [1-2], and also on topographical features on the nanoscale [3-12] such as, for example, grooves a few tens of nanometres across. We culture cells on isotropic, aligned and patterned substrates based on multiwall carbon nanotubes and hence with nanoscale surface features. The cells adhere to and grow on all substrates, and on the aligned substrate, the cells align strongly with the axis of the bundles of the multiwall nanotubes. The substrates are made using straightforward physical chemistry techniques, thus they can easily and cheaply be scaled up to produce substrates for tissue engineering.

The response of cells to surfaces they contact is mediated via clusters of proteins called focal adhesions. Nanoscale topography affects the formation of these focal adhesions and hence cell behaviour. This should be unsurprising to us. In our bodies, cells are surrounded by and interact with a complex three-dimensional extra-cellular matrix (ECM) environment which has nanoscale structure. The ECM provides physical cues which affect cell behaviour and hence the formation of functional tissues from cells. Thus, materials with controlled nanoscale topography can be used to mimic ECM features and to direct cell behaviour. These materials are required for successful tissue engineering.

The topography of the substrate can be smooth, rough (i.e., disordered) [13] or with a periodically varying height [14]. The substrate can also be isotropic or anisotropic. By rough we mean that the height varies but not with a simple repeating pattern, although it may have a characteristic lengthscale. An isotropic substrate is the same in all directions and so provides no cue to orient the cells, while on an anisotropic substrate the cells may align along a particular axis of the substrate. Anisotropic substrates comprised of grooves and ridges have been systematically studied and found to influence cell migration, elongation and alignment along the directions of the grooves and ridges, a phenomenon known as contact guidance [15-18]. 
Also, for cells on grooves and ridges, cell behaviour has been shown to be sensitive to substrate features such as the groove width, depth, and the pitch of the pattern [19-21]. In the work discussed above, the substrate pattern is on lengthscales varying from a few times larger than a single protein to approximately the size of a focal adhesion $(\approx$ micrometre $)$.

Substrates can also be patterned on the lengthscale of a cell. For example, O’Neill et al. [22] and Chen et al. [23] showed that a substrate with islands that promoted adhesion surrounded by a surface that the cells could not adhere to, could directly control the size and shape, and hence the behaviour of cells. Subsequent work has seen dramatic effects on the cell cytoskeleton [22, 24-25], and varied the substrate geometry, for example Hidai et al. [26] studied cells on cylindrical fibres that had diameters comparable to the cell size. Both NIH 3T3 and MDCK cells shown cell morphologies on these fibres that are very different from the morphology on flat substrates. The organisation of the cell's actin bundles is very different for cells growing on fibres than it is for cells growing on a flat substrate. Edwards et al. [27] examined NR6 mouse fibroblast cells at on a tubular micro-scale scaffold composed of a glass rod wrapped with a 9 ply multiwalled carbon nanotubes yarn. They also looked at cells on an electrospun polymer/MWNT composite. In addition, recent work by Jeon et al. [28] has looked at grids of varying aspect ratio.

It is important to note that many different cell types have been studied in work on cellsubstrate interactions, for example, fibroblasts [6, 29-32], epithelial cells [15, 33], cardiac cells [34], osteoblasts [35-37] and neurite cells [38]. The response of a cell to a substrate will in general depend on the cell type as well as the substrate, although a number of different cell types have all been shown to align with the grooves of aligned substrate so alignment may a generic to many types of mammalian cell [5]. The substrates themselves have been produced via a number of methodologies, such as photolithography [39-40], microstamping [41-42], hot embossing [43], stencil patterning [44], nanoimprint lithography [45], electrospinning [27, 46] and phase separation $[37,47]$. 
Carbon nanotubes have a number of advantages for substrates for cell growth. They are inert, strong, electrically conductive, naturally have nanoscale topography and can easily be patterned on scales of micrometres and above. They have been shown to be non-toxic and biocompatible, for example Mattson et al. [48] grew rat hippocampal neurons for up to 8 days on nanotube substrates. They can also easily be functionalised to change their surface chemistry, for example by attaching molecules with biological activity such as DNA, hyaluronic acid and chitosan [49]. For example, Hu et al. [50] found that the rat hippocampal neurons have more growth cones with extensive branching and longer neurites on positively charged MWNTs. Their electrical conductivity may be an especially useful property for neural tissue engineering $[48,50]$. Other studies have shown that carbon-nanotube based scaffolds support the growth of other cells types, for example fibroblasts [31-32] and osteoblasts [36, 51-53].

Here we study the effect on cells of two types of carbon-nanotube based substrates: one isotropic substrate and one aligned. Both are made with multiwall nanotubes (MWNTs). Nerve cells have been studied on our aligned substrates [54] in earlier work [10]. We have found that the nanoscale topography of these substrates influences cell behaviour. Cell alignment is required in many tissues, e.g. liver and skin. Our Chinese Hamster Ovary (CHO) cells align strongly with the nano-scale grooves of our aligned substrates. This agrees with previous work on CHO cells by Rebollar et al. [55], who reported the alignment of CHO cells with a periodic surface nanostructure. $\mathrm{CHO}$ cells were chosen due to the fact that they are well studied, easy to culture, and so is an ideal cell type [56-57] for initial experiments on a novel substrate. We are currently working on other cell types, including primary cells, on our substrates.

Recent work by Lima et al. [58] has shown that the MWNT sheets we use as our aligned substrates can be spun into a range of yarns with functional guest particles. These functionalised yarns could provide an aligned three-dimensional substrate for cell growth, with incorporated growth factors, mineral particles for osteoblasts, etc. Here we study simple threads of our MWNTs and show that cells adhere to, and stretch along these threads. Future work on 
our MWNT-based materials could build on the work here to look at more complex threedimensional geometries and functionalised substrates.

\section{Materials and methods}

\subsection{Preparation of aligned MWNT films}

MWNT forests were produced via solid-state catalytic chemical vapor deposition (CVD), with catalyst and buffer layers deposited on silicon wafers (as the catalyst support). Acetylene gas was used as the carbon source. TGA data reports the purity of the MWNTs utilized at $\sim 3 \mathrm{wt} \%$ catalytic contaminants. The diameter of the MWNTs is approximately $10 \mathrm{~nm}$, and the forest heights averaged $185 \mu \mathrm{m}$. MWNT sheets were drawn from the sidewall of the forest via sharpedge compression utilizing a standard straight-edge razor blade (with a width of $4 \mathrm{~cm}$ and at an approximate $45^{\circ}$ angle to the forest) and subsequently dragged outwards to obtain an anisotropic aerogel sheet of aligned MWNT bundles [54]. Fibril branching continues throughout the sheet, thereby making a laterally extended, interconnected fibril network. These aerogel sheets are ultra-light, transparent, and electrically conductive, having an areal density of approximately 1-3 $\mu \mathrm{g} / \mathrm{cm}^{2}$. The MWNT sheets are highly aligned, Zhang et al. [54] applied Raman spectroscopy and reported a polarisation degree of between 0.69 and 0.75 .

The aerogel sheets were then applied to glass substrates for shipping from UTD to Surrey. During the application process, the sheets were densified along the alignment of the nanotubes with 2-propanol and allowed to air-dry. The sheets produced are similar to that shown in Fig. S2 of the work of Zhang et al. [54]. At Surrey, before use in cell culture, a sheet was transferred from the glass substrate to a cover glass. This was done as follows: Water was dropped onto the substrate using a pipette, which caused the MWNT bundles to lift off the substrate. A cover glass was then slipped under the bundles. The cover glass with the MWNT sheet was then dried in an oven at $60-70^{\circ} \mathrm{C}$ for approximately $12 \mathrm{hr}$. We believe that this procedure will further densify the MWNT sheets, compare the density of bundles in the SEMs of figure 1(B) and (C) 
with the density in figure S2 [54]. The procedure also introduces some variability in the local density of the MWNT bundles. This non-uniformity can be seen in the optical image of our sheets, figure 1(D), where the darker curves are larger denser MWNT bundles. It should also be borne in mind that whenever a liquid is added to the substrate (e.g., when cells are added) there is movement of the MWNT bundles due to the fact that the bundles are not bonded to the glass.

In addition to the films made with a single sheet of aligned MWNTs, we also created crossed MWNT substrates by simply laying one aligned MWNT sheet down on another such that the top and bottom sheets are perpendicular.

\subsection{Preparation of isotropic MWNT films}

The MWNTs for making isotropic buckypaper were obtained from Nanocyl (95\% purity). They are synthesized via catalytic chemical vapour deposition (CVD). A MWNT suspension was prepared in chloroform at a concentration of $0.01 \mathrm{mg} / \mathrm{ml}$, i.e., $5 \mathrm{mg}$ of MWNTs in $500 \mathrm{ml}$ of chloroform. The MWNT suspension in chloroform was sonicated using a bath sonicator (Fisherbrand) for 100 minutes. $400 \mathrm{ml}$ of the stock suspension was then centrifuged at 25,000 g for one hour, half the supernatant was then drawn off and this was centrifuged again at 25,000 $\mathrm{g}$ for one hour. This process of drawing off half the supernatant and centrifuging it was then repeated three more times (i.e., a total of four times). $20 \mathrm{ml}$ of the resulting suspension was filtered through a nitrocellulose membrane (Millipore) using a rotary pump (1425 rpm, Edwards). The buckypaper was then kept in an oven at the temperature of $75^{\circ} \mathrm{C}$ for $12 \mathrm{hr}$ to remove the chloroform. To remove the buckypaper from the membrane, the nylon membrane was dissolved in acetone, using a number $(\sim 5)$ of volumes of acetone. The membrane was immersed in $10 \mathrm{ml}$ of acetone for 30 minutes and then the acetone was replaced by another fresh volume of $10 \mathrm{ml}$. This process was repeated with a total of around 5 volumes of acetone to make sure that the membrane was completely removed. The buckypaper, now floating in acetone, was attached to a cover glass. The buckypaper and glass were then annealed at $180^{\circ} \mathrm{C}$

for $12 \mathrm{hr}$ to get rid of any residual chloroform and acetone. AFM and SEM images of the 
substrates are shown in figure 2. Both types of substrates were characterised using SEM (Hitachi, S4000, for figure 1(B) and figure 2(B), and a Zeiss SIGMA Advanced Analytical SEM for figure 1(C)), and AFM (NT-MDT). The hydrophilicity of the prepared substrates was measured using contact angle measurement of 1 l water drops (Easy Drop, Krüss GmbH, Germany).

\subsection{Cell Culture}

Chinese Hamster Ovary (CHO) cells (Cat No: 85050302) with epithelial morphology were purchased from European Collection of Cell Cultures (ECACC). Established protocols by ECACC (www.hpacultures.org.uk/collections/ecacc.jsp) for the culture and passage of CHO cells were followed. Cells were cultured in tissue culture flasks $\left(75 \mathrm{~cm}^{2}\right)$ in a humidified atmosphere at $37^{\circ} \mathrm{C}$ with $5 \% \mathrm{CO}_{2}$ (by volume). The cells were grown in F-12 Ham's medium (Gibco, Invitrogen) supplemented with $10 \%$ by volume fetal bovine serum (Gibco, Invitrogen) and $1 \%$ (by volume) of antibiotics solution (penicillin/streptomycin). Cultures were passaged every 48 hours by a dilution factor of $1 / 6$ or every 72 hours by a dilution factor of $1 / 7$. Passaging was done by first rinsing with sterile phosphate buffered saline (PBS) and then detaching the cells by incubating with $2.5 \%$ of trypsin-EDTA (Gibco, Invitrogen) solution for 3-5 min. For seeding samples, culture flasks with $80 \%$ confluency of $\mathrm{CHO}$ cells were trypsinized, washed and suspended in fresh media. The suspension of cells was then diluted with cell growth media to the desired cell concentration following a cell count using the trypan blue exclusion method and a haemocytometer. For all microscopy and imaging experiments, cell suspensions with density of $1-1.5 \times 10^{3}$ cells per ml per well were added to six-well plate polystyrene Petri dishes containing prepared substrates and incubated for 20 hours. Prepared substrates were exposed to ultraviolet (UV) light for 30 minutes to sterilize them before use in cell experiments. In order to observe if the MWNT sheets used in this study have any toxic effect on cells, cells were allowed to grow on the substrates until about $90 \%$ confluence. 
The fixing and staining of cells was done as follows. The cells-seeded substrate was washed with PBS and fixed with 4\% paraformaldehyde in PBS for 20 min. After fixation, the samples were rinsed gently with PBS and permeabilized with $0.1 \%$ non-ionic surfactant, Triton $\mathrm{X}$ (Sigma) in PBS for 5 min. Samples were then thoroughly washed with PBS and stained. Samples were incubated with AlexaFluor phalloidin (Molecular Probes) at concentration of 0.2 $\mu \mathrm{M}$ for $30 \mathrm{~min}$ at room temperature. After rinsing with $\mathrm{PBS}$, samples were subsequently incubated with DRAQ5 (BD Biosciences Limited) at a concentration of $5 \mu \mathrm{M}$ for $10 \mathrm{~min}$ at room temperature. Stained cells were then mounted with a coverslip in mounting medium (Vectashield, Vector Laboratories, Inc., Burlingame, CA) and sealed with nail varnish. Doublelabelled samples were examined via confocal microscopy (Zeiss LSM 510 META).

The confocal microscopy and image acquisition was done as follows. Imaging was conducted by using a Zeiss LSM 510 META laser scanning confocal microscope. For the double stained cells, AlexaFluor phalloidin (actin stain) was excited with the argon laser line of $488 \mathrm{~nm}$ and DRAQ5 (nucleus counterstain) with the helium-neon laser line of $633 \mathrm{~nm}$. The emission signals passed through the 505-530 nm and 649-799 nm filters respectively. All images were captured with either a Plan-Apochromat 40x or 63x/1.4 NA oil immersion phase objective and collected in multichannel mode. A multi track configuration was used in order to minimize any bleedthrough effect from the different channel. Pinholes were set at 1 Airy unit (AU), which corresponds to an optical slice of $0.9 \mathrm{~nm}$ for both channels. All confocal data sets were of frame size 512 by 512 pixels, scan zoom of 1 and line averaged four times. All images were processed by using the Zeiss LSM browser.

2.4 Quantitative analysis of CHO cell alignment and morphology 
We wished to quantify the orientational alignment of cells on our aligned MWNT substrates, and wanted to do so in a simple way that was as parameterfree as possible. To do this we needed to unambiguously determine an axis of a single cell. This was done by taking a confocal image of the cells that combines the red (nucleus staining) and green (actin staining) channels and thresholding it to produce a binary black/white bitmap that was white over almost all the area of all the cells present, and black elsewhere. We discovered that varying the threshold within reasonable limits had little effect on our results. We then identified each cell or cluster of touching cells, as being a set of connected white pixels. The (2D) moment-of-inertia tensor was then calculated for each cell and diagonalised. The eigenvector corresponding to the smaller eigenvalue is taken to define the long axis of the cell. The orientation angle $\theta$ is then the angle this eigenvector makes with the horizontal in the images of figure 3, which is the direction of alignment of the MWNTs. The elongation or aspect ratio of the cell is taken to be the square root of the ratio of the large to the small eigenvalue. It is the square root as the eigenvalues scale as length squared. The size of a cell or cell cluster is its number of pixels times the area of a pixel, which is $0.0866 \mu \mathrm{m}^{2}$ at $40 \mathrm{x}$ magnification. The analysis was performed for 6 images for the aligned MWNT substrates, 4 images for cells on the crossed MWNT substrates, and 5 images for the control glass substrates. All images used were confocal images at 40x magnification with both DRAQ5 and phalloidin staining.

\subsection{Analysis of biocompatibility of the MWNTs}

This was assessed by allowing the cells to grow to approximately $90 \%$ confluence, i.e., until they covered approximately $90 \%$ of the area of the substrate surface. This took 43 hours total time of the cells on the substrate: the cells were cultured for 20 hours, the growth medium was then changed and the cells were cultured for an additional 23 hours. The cells were fixed and stained with both phalloidin and DRAQ5. Images obtained with the $63 \mathrm{x}$ objective were then 
analysed using the image analysis software Image $\mathbf{J}$ from the United States National Institutes of Health [59], in order to quantify the amount of cells that have adhered to and grown on the substrate. The quantification was done by evaluating the fraction of image areas that are occupied by cells. Any toxicity of the MWNT over the approximately 43 hours will result in the cells not growing as rapidly and so covering a smaller fraction of the substrate surface. Each image of doubly stained cells was thresholded using the same threshold for all images. This was done for 21 images obtained from three aligned MWNT substrates and for 11 images from two control glass substrates. These substrates were studied in two independent experiments. The results were then analyzed using Origin 6.1 (OriginLab, USA).

\section{Results and discussion}

\subsection{Substrate characterisation}

We use two types of MWNT sheets as substrates: isotropic and aligned sheets. Their physical and chemical properties were characterised by AFM (tapping mode), SEM, and contact angle measurement. The root mean square (RMS) roughness of the sheets was obtained via AFM scanning (tapping mode) of an area of $50 \times 50 \mu \mathrm{m}$; see figures 1 and 2 . The isotropic and aligned sheets have similar RMS roughnesses of 60 and $51 \mathrm{~nm}$, respectively. The isotropic sheets are rather hydrophobic, while the aligned sheets are moderately hydrophilic. The contact angles of water droplets are $123 \pm 5^{\circ}$ on the isotropic film, and $73 \pm 5^{\circ}$ on the aligned films. We believe that the lower contact angle of the aligned substrate is due to interaction of water with the (hydrophilic) glass underneath the MWNT bundles. Also, we note that the MWNT bundles will move when water is dropped onto the sheets. They are not stuck down and the air/water interface (of a water droplet or of the growth medium with cells) can exert significant forces, as can cells themselves. Thus MWNT bundles may move due to the force of the growth medium/air interface when the growth medium flows across the substrate, and also 
possibly when the cells themselves pull on the bundles. Thus, even with an initial almost complete coverage of the glass by MWNTs (seen in figure 1) it is possible that glass may be exposed (as our contact angle measurements suggest). Then parts of the cell may contact and hence interact with the glass. As the MWNT bundles can move our substrates should therefore be thought of as plastically deforming when the cells adhere to them and bind to and pull on the MWNT bundles. It is known that cells can sense and alter their behaviour in response to how compliant the substrate is $[1-2,60]$. So, the compliance of our substrates may be contributing to the response of the cells that we observe. Cells may behave differently on substrates that have similar topography to ours but that are rigid.

\subsection{CHO cells on substrates with nanoscale topography}

Twenty hours after seeding the cells onto control substrates, isotropic and aligned sheets of MWNTs, we stained the cells and studied them using confocal microscopy (figure 3) and AFM (figure 4). We also studied their alignment and elongation quantitatively (figure 5). The cells adhered and grew on both MWNT-based substrates but rather better on the aligned MWNT sheets. Adhesion was poor on the buckypaper substrates, few cells adhere to these substrates and so we see a low coverage in figure 3(D). However, there is no evidence of MWNT toxicity as the cells grow well on the aligned-MWNT substrates (see toxicity study below). Thus our aligned substrates are highly suitable for cell culturing but our buckypaper is probably not suitable. If indeed on the aligned-MWNT substrates the cells are partly interacting with the glass, and partly with the MWNTs (see section 3.1), then it may be that adhesion is promoted by this mixture of MWNTs and glass but is inhibited if the cells interact only with MWNTs, as they do on the buckypaper.

In figure 3 we show images where we have merged actin staining (green) and nucleus staining (red) confocal fluorescence images, and a DIC image. In figure 4 we show AFM images of single cells on the substrate. Note that the cell on the aligned substrate, figure 4(D) 
and (E), is highly elongated along the direction of the MWNT bundles; unlike the microscopy images, this AFM image is not typical, most cells are not highly elongated. See the confocal image in figure 3(B) for typical cell morphologies. These morphologies are aligned with the direction of the MWNTs but not highly elongated; see our quantitative analysis in figure 5. The cells on our buckypaper substrates are mostly round in shape as shown in figure 4 (C) and (D), but the cells on glass have spread and elongated in all directions, with very few of them showing circular morphology (figure 4 (A) and (B)). Most cells attached and spread well on bare glass, with characteristic actin (green) fibres clearly seen in figure 3 (A).

If we compare the $\mathrm{CHO}$ cells on the aligned MWNT substrate with those on the glass control we see three main differences: 1) many cells on the MWNT substrates align with the MWNT bundles; 2) some elongate strongly along the axis defined by this groove (the horizontal axis in figure 3 and vertical in figure 4); 3) the cells cluster together less than on the control substrate compare figure 3(A) with 3(B).

The most striking observation is the alignment of the cells with the MWNT bundles. On the aligned sheet, $80 \%$ of the cells have their long axis within $30^{\circ}$ of the direction defined by the MWNT bundles. Cells on the aligned-MWNT substrates are represented by red squares in figure $5.80 \%$ of them lie with $30^{\circ}$ of the horizontal in figure 5 . The horizontal is the direction of the MWNTs. Figure 5 is a polar plot and the distance from the origin is the elongation minus one, so a perfectly circular cell would be at the origin, and long and thin cells are far from the origin. Cells with their long axis marked on them are shown in the inset of figure 5. It is clear from figure 5 that cells detect and respond strongly to the anisotropy of the surface. The cells on glass (blue circles) are clustered near the origin indicating that they are approximately circular, and they are uniformly distributed in angle. By contrast on the aligned-MWNT substrate some cells are highly elongated and most are aligned with the MWNT direction. Our substrates hold promise as use as scaffolds for tissue engineering applications where tissues 
with aligned cells are required. Most tissues in the body are composed of cells that are not only aligned but polar. We did not attempt to look for polarization in our cells.

A minority of the cells are not only aligned with the MWNT bundles, they also stretch along the bundles: $16 \%$ of the cells on our aligned sheets have an anisotropy ratio of over $3: 1$. This ratio is defined as the square root of the ratio of the large to the small eigenvalue of the moment of inertia tensor. Note that very few cells/cell clusters have ratios greater than 3 on our control substrates, see figure 5. However, many cells align without elongating, so stretching a large amount along the bundles is clearly not required in order to align with them. Note the large numbers of cells in figure 5 with small angles, $\theta$, to the bundles but with elongations of around 2 or less. These small elongations are comparable to the typical elongations found on the glass control substrate. On the crossed MWNT substrate, cells did not align significantly with either the top or the bottom sheet, see figures 3 and 5 .

The reduced clustering of the cells is apparent from the reduced mean area of the cells/cellclusters on the aligned MWNT substrate. This area is only $110 \mu \mathrm{m}^{2}$ as opposed to $520 \mu \mathrm{m}^{2}$ on the glass substrate. On the aligned MWNT substrate a large majority of the CHO cells are single cells, whereas most are in clusters on the glass surface, which increases the average area per cell cluster. They also cluster less on our isotropic buckypaper, but this may be associated with the lower cell density on these substrates. To summarise, we have found that the nanoscale topographical features of our substrates significantly influence aspects of cell behaviour such as alignment, morphology and cell-to-cell adhesion.

\subsection{CHO cells on patterned substrates}

CHO cells respond strongly to the nanoscale structure of our substrates, and so control over this nanoscale topography allows control over the cells. Therefore, we varied this nanoscale topography in space over lengthscales comparable to the size of the cell. We produced patterns by forming gaps in the top MWNT sheet in one of our crossed MWNT substrates (figure 6), and by putting single threads of bundles of MWNTs on glass (figure 7). We found (figure 6) 
that when the gap was comparable in size to the cells, cells bridged this gap. Also, in figure 7 we show AFM images of a CHO cell on a thread of MWNTs. This thread was obtained from one of our aligned sheets. The thread was attached to the cover glass by nail polish at each end. The morphology is clear in the phase image (see figure 7(B)). From the image, it seems that the cell adheres to the nanotube thread and elongates along it. Both the bridging behaviour and the stretching along the thread shows that large scale $(\sim 10 \mu \mathrm{m})$ features also influence cell morphology.

We note that the AFM images of figure 7 are selected to show cells elongating along a thread. Cells adhere both to the thread and to the bare cover glass around the thread. Of the cells that adhere to the thread, some align and a few of them spread over the thread (but do not elongate).

\subsection{Short term toxicity study}

We performed a short term toxicity study in order to test the biocompatibility of our prepared substrates. This was done by allowing cells to grow until they covered approximately $90 \%$ of the area of the substrate surface. Cells on both substrates were then fixed and imaged using confocal microscopy. We did not study the isotropic buckypaper as the CHO cells adhered less well to these substrates. The fraction of the substrate covered by cells was quantified using ImageJ software. Any toxicity of the MWNTs over the 43 hours will result in the cells not growing as rapidly and so a covering a smaller fraction of the substrate surface.

In figure 8 we show the fraction of the substrate area covered by the cells, for both our aligned MWNT substrates and on control substrates. The coverages are $77 \pm 7 \%$ on the glass control substrate and $65 \pm 9 \%$ on the aligned MWNT substrate. The error bars for the coverage of the two substrates overlap so the cells appear to grow as well on the aligned MWNT substates as on the control substrate. We did not observe any significant differences in the 
morphology of the cell's nucleus (stained with DRAQ5) between the two substrates. We did not attempt to perform a long term toxicity study and so can draw no conclusions about longterm toxicity. However over the 43 hours of this study there is no evidence for any toxicity of the MWNTs.

\section{Conclusion}

Here we have shown that the widely studied CHO cells can be grown on both isotropic and aligned multi-wall carbon nanotube films. The films have features tens of nanometres high and with a comparable pitch. On the aligned sheet, almost all cells orient along with their longest axis along the bundles; $80 \%$ of the cells have their long axis within $30^{\circ}$ of the axis of the MWNT bundles. However, only a few cells stretch out and become long and thin; only 16\% have an anisotropy ratio of over 3:1. Thus, from the results of our quantitative analysis, it is clear that alignment can and does occur without a pronounced morphology change. Interestingly, although the cell morphology does not change significantly for most cells, the cells cluster much less on our aligned substrates than on our control glass substrates. Thus we have demonstrated control over clustering using nanoscale topography, but further work would be needed to see whether nanoscale topography can also enhance clustering. We expect that optimal tissue-engineering substrates would generally be those that encourage clustering of the cells.

Thus, surface roughnesses of order tens of nanometres are enough to change the cell behaviour. Teixiera et al. [21] studied corneal epithelial cells on substrates with grooves a little larger than our MWNT bundles but still nanoscale. They imaged the protein vinculin, a component of focal adhesions, and found that in their cells focal adhesions aligned with the grooves. Although we have not imaged focal adhesions here, we speculate that in our $\mathrm{CHO}$ cells the focal adhesions may be lining up with the MWNT bundles and this aligns the bundles of actin inside the cell which in turn aligns the entire cell. Estévez et al. [61] also showed how substrate topography directs the cell's focal adhesions and cytoskeleton. 
Other previous work has studied many cell types on a wide range of substrates with nanometre scale patterning. It has been shown that this patterning affects cell adhesion, spreading and morphology. This includes earlier work with different cell types on aligned substrates very similar to those used here [54]. Most (but not all) cell types align themselves with the substrate pattern on aligned substrates; see the recent review of Bettinger et al. [5] Rebollar et al. [55] have shown that $\mathrm{CHO}$ cells aligned on substrates with periodic grooves. Their grooves have a period somewhat larger than the size of our MWNT bundles but the period was less than $0.5 \mu \mathrm{m}$. Thus, based on the work presented here and that of Galvan-Garcia et al. [10], we would expect cells of most cell types to align themselves on our aligned substrates. Our substrates should therefore be useful for producing tissues in which it is necessary to align the cells.

We also looked at patterned substrates, i.e. substrates where the MWNT coverage was nonuniform over length scales of order the size of the cell or larger. We found with our MWNT threads that a cell could adhere to, and stretch along the thread, see figure 7, forming a strongly elongated morphology not found for cells on conventional glass substrates. The cell morphology is similar to that found on fibres made via two-photon polymerisation [26]. However our substrates are made from MWNTs using simple physical chemistry techniques. No lithography or photopolymerisation is required. Furthermore recent work by Lima et al. [58] has produced complex yarns and knots which can include large amounts of a guest species. Thus our work here showing that the MWNT sheets are biocompatible and that they align the cells is just the beginning, future work could consider more complex three-dimensional geometries such as twisted yarns and knots, and also study functionalised substrates.

\section{Acknowledgements}

CACA acknowledges the Malaysian Ministry of Higher Education (MOHE) and the Universiti Putra Malaysia for providing financial support for her PhD studies. PA acknowledges the Royal Thai Government for his PhD funding. RPS and ABD acknowledge financial support from $\operatorname{EPSRC}(\mathrm{EP} / \mathrm{G} 060878 / 1)$. 


\section{References:}

[1] Lo C M, Wang H B, Dembo M and Wang Y L 2000 Cell movement is guided by the rigidity of the substrate Biophysical Journal 79 144-52

[2] Saez A, Ghibaudo M, Buguin A, Silberzan P and Ladoux B t 2007 Rigidity-driven growth and migration of epithelial cells on microstructured anisotropic substrates Proceedings of the National Academy of Sciences 104 8281-6

[3] Chung T-W, Liu D-Z, Wang S-Y and Wang S-S 2003 Enhancement of the growth of human endothelial cells by surface roughness at nanometer scale Biomaterials 24 465561

[4] Lampin M, Warocquier-Clérout R, Legris C, Degrange M and Sigot-Luizard M F 1997 Correlation between substratum roughness and wettability, cell adhesion, and cell migration Journal of Biomedical Materials Research 36 99-108

[5] Bettinger C J, Langer R and Borenstein J T 2009 Engineering Substrate Topography at the Micro- and Nanoscale to Control Cell Function Angewandte Chemie-International Edition 48 5406-15

[6] Dalby M J, Childs S, Riehle M O, Johnstone H J H, Affrossman S and Curtis A S G 2003 Fibroblast reaction to island topography: changes in cytoskeleton and morphology with time Biomaterials 24 927-35

[7] Heydarkhan-Hagvall S, Choi C-H, Dunn J, Heydarkhan S, Schenke-Layland K, MacLellan W R and Beygui R E 2007 Influence of Systematically Varied Nano-Scale Topography on Cell Morphology and Adhesion Cell Communication and Adhesion 14 181-94

[8] Clark P, Connolly P, Curtis A S, Dow J A and Wilkinson C D 1990 Topographical control of cell behaviour: II. Multiple grooved substrata Development 108 635-44

[9] Haq F, Anandan V, Keith C and Zhang G 2007 Neurite development in PC12 cells cultured on nanopillars and nanopores with sizes comparable with filopodia International Journal of Nanomedicine 2 107-15

[10] Galvan-Garcia P, Keefer E W, Yang F, Zhang M, Fang S, Zakhidov A A, Baughman R $\mathrm{H}$ and Romero M I 2007 Robust cell migration and neuronal growth on pristine carbon nanotube sheets and yarns Journal of Biomaterials Science-Polymer Edition 18 124561

[11] Tutak W, Park K H, Vasilov A, Starovoytov V, Fanchini G, Cai S Q, Partridge N C, Sesti F and Chhowalla M 2009 Toxicity induced enhanced extracellular matrix production in osteoblastic cells cultured on single-walled carbon nanotube networks Nanotechnology 20255101

[12] Tutak W, Chhowalla M and Sesti F 2010 The chemical and physical characteristics of single-walled carbon nanotube film impact on osteoblastic cell response Nanotechnology 21315102

[13] Guénard V, Valentini R F and Aebischer P 1991 Influence of surface texture of polymeric sheets on peripheral nerve regeneration in a two-compartment guidance system Biomaterials 12 259-63 
[14] Hunt J A, Williams R L, Tavakoli S M and Riches S T 1995 Laser surface modification of polymers to improve biocompatibility Journal of Materials Science: Materials in Medicine 6 813-7

[15] Teixeira A I, Abrams G A, Bertics P J, Murphy C J and Nealey P F 2003 Epithelial contact guidance on well-defined micro- and nanostructured substrates J Cell Sci 116 $1881-92$

[16] Clark P, Connolly P, Curtis A S, Dow J A and Wilkinson C D 1991 Cell guidance by ultrafine topography in vitro J Cell Sci 99 73-7

[17] Dunn G A and Brown A F 1986 Alignment of fibroblasts on grooved surfaces described by a simple geometric transformation J Cell Sci 83 313-40

[18] Fujita S, Ohshima M and Iwata H 2009 Time-lapse observation of cell alignment on nanogrooved patterns Journal of The Royal Society Interface 6 S269-S77

[19] Fraser S A, Ting Y-H, Mallon K S, Wendt A E, Murphy C J and Nealey P F 2008 Submicron and nanoscale feature depth modulates alignment of stromal fibroblasts and corneal epithelial cells in serum-rich and serum-free media Journal of Biomedical Materials Research Part A 86A 725-35

[20] Andersson A-S, Olsson P, Lidberg U and Sutherland D 2003 The effects of continuous and discontinuous groove edges on cell shape and alignment Experimental Cell Research 288 177-88

[21] Teixeira A I, McKie G A, Foley J D, Bertics P J, Nealey P F and Murphy C J 2006 The effect of environmental factors on the response of human corneal epithelial cells to nanoscale substrate topography Biomaterials 27 3945-54

[22] Ireland G W, Doppinghepenstal P, Jordan P and Oneill C 1987 Effect of Patterned Surfaces of Adhesive Islands on the Shape, Cytoskeleton, Adhesion and Behavior of Swiss Mouse 3t3 Fibroblasts Journal of Cell Science 19-33

[23] Chen C S, Mrksich M, Huang S, Whitesides G M and Ingber D E 1997 Geometric control of cell life and death Science 276 1425-8

[24] Oakley C and Brunette D M 1993 The Sequence of Alignment of Microtubules, Focal Contacts and Actin-Filaments in Fibroblasts Spreading on Smooth and Grooved Titanium Substrata Journal of Cell Science 106 343-54

[25] Wojciakstothard B, Madeja Z, Korohoda W, Curtis A and Wilkinson C 1995 Activation of Macrophage-Like Cells by Multiple Grooved Substrata - Topographical Control of Cell Behavior Cell Biology International 19 485-90

[26] Hidai H, Jeon H, Hwang D J and Grigoropoulos C P 2009 Self-standing aligned fiber scaffold fabrication by two photon photopolymerization Biomedical Microdevices 11 $643-52$

[27] Edwards S L, Church J S, Werkmeister J A and Ramshaw J A M 2009 Tubular microscale multiwalled carbon nanotube-based scaffolds for tissue engineering Biomaterials 30 1725-31

[28] Jeon H, Hidai H, Hwang D J, Healy K E and Grigoropoulos C P 2010 The effect of micronscale anisotropic cross patterns on fibroblast migration Biomaterials 31 4286-95 
[29] Berry C C, Campbell G, Spadiccino A, Robertson M and Curtis A S G 2004 The influence of microscale topography on fibroblast attachment and motility Biomaterials 25 5781-8

[30] Dalby M J, Giannaras D, Riehle M O, Gadegaard N, Affrossman S and Curtis A S G 2004 Rapid fibroblast adhesion to $27 \mathrm{~nm}$ high polymer demixed nano-topography Biomaterials 25 77-83

[31] Yuen F L Y, Zak G, Waldman S D and Docoslis A 2008 Morphology of fibroblasts grown on substrates formed by dielectrophoretically aligned carbon nanotubes Cytotechnology 56 9-17

[32] Yun Y H, Dong Z Y, Tan Z Q, Schulz M J and Shanov V 2009 Fibroblast cell behavior on chemically functionalized carbon nanornaterials Materials Science \& Engineering C-Biomimetic and Supramolecular Systems 29 719-25

[33] Karuri N W, Liliensiek S, Teixeira A I, Abrams G, Campbell S, Nealey P F and Murphy C J 2004 Biological length scale topography enhances cell-substratum adhesion of human corneal epithelial cells Journal of Cell Science 117 3153-64

[34] Deutsch J, Motiagh D, Russell B and Desai T A 2000 Fabrication of microtextured membranes for cardiac myocyte attachment and orientation Journal of Biomedical Materials Research 53 267-75

[35] Zhao G, Raines A L, Wieland M, Schwartz Z and Boyan B D 2007 Requirement for both micron- and submicron scale structure for synergistic responses of osteoblasts to substrate surface energy and topography Biomaterials 28 2821-9

[36] Zanello L P, Zhao B, Hu H and Haddon R C 2006 Bone cell proliferation on carbon nanotubes Nano Letters 6 562-7

[37] Jell G, Verdejo R, Safinia L, Shaffer M S P, Stevens M M and Bismarck A 2008 Carbon nanotube-enhanced polyurethane scaffolds fabricated by thermally induced phase separation Journal of Materials Chemistry 18 1865-72

[38] Rajnicek A M, Britland S and McCaig C D 1997 Contact guidance of CNS neurites on grooved quartz: influence of groove dimensions, neuronal age and cell type Journal of Cell Science $1102905-13$

[39] Britland S, Morgan H, WojiakStodart B, Riehle M, Curtis A and Wilkinson C 1996 Synergistic and hierarchical adhesive and topographic guidance of BHK cells Experimental Cell Research 228 313-25

[40] Molnar P, Wang W S, Natarajan A, Rumsey J W and Hickman J J 2007 Photolithographic patterning of $\mathrm{C} 2 \mathrm{C} 12$ myotubes using vitronectin as growth substrate in serum-free medium Biotechnology Progress 23 265-8

[41] Takii Y, Kaji H, Matsue T and Nishizawa M 2004 Microstamp-based micromachining for modulation of growth of cultured neuronal cells Jsme International Journal Series C-Mechanical Systems Machine Elements and Manufacturing 47 956-61

[42] Chang J C, Brewer G J and Wheeler B C 2003 A modified microstamping technique enhances polylysine transfer and neuronal cell patterning Biomaterials 24 2863-70

[43] Charest J L, Eliason M T, Garcia A J and King W P 2006 Combined microscale mechanical topography and chemical patterns on polymer cell culture substrates Biomaterials 27 2487-94 
[44] Folch A, Jo B H, Hurtado O, Beebe D J and Toner M 2000 Microfabricated elastomeric stencils for micropatterning cell cultures Journal of Biomedical Materials Research 52 346-53

[45] Chou S Y, Krauss P R and Renstrom P J 1996 Imprint lithography with 25-nanometer resolution Science 272 85-7

[46] Prabhakaran M P, Venugopal J R and Ramakrishna S 2009 Mesenchymal stem cell differentiation to neuronal cells on electrospun nanofibrous substrates for nerve tissue engineering Biomaterials 30 4996-5003

[47] Riehle M O, Dalby M J, Johnstone H, MacIntosh A and Affrossman S 2003 Cell behaviour of rat calvaria bone cells on surfaces with random nanometric features Materials Science \& Engineering C-Biomimetic and Supramolecular Systems 23337 40

[48] Mattson M P, Haddon R C and Rao A M 2000 Molecular functionalization of carbon nanotubes and use as substrates for neuronal growth Journal of Molecular Neuroscience 14 175-82

[49] Thompson B C, Moulton S E, Gilmore K J, Higgins M J, Whitten P G and Wallace G G 2009 Carbon nanotube biogels Carbon 47 1282-91

[50] $\mathrm{Hu} \mathrm{H}$, Ni Y C, Montana V, Haddon R C and Parpura V 2004 Chemically functionalized carbon nanotubes as substrates for neuronal growth Nano Letters 4 50711

[51] Elias K L, Price R L and Webster T J 2002 Enhanced functions of osteoblasts on nanometer diameter carbon fibers Biomaterials 23 3279-87

[52] Akasaka T, Yokoyama A, Matsuoka M, Hashimoto T, Abe S, Uo M and Watari F 2009 Adhesion of human osteoblast-like cells (Saos-2) to carbon nanotube sheets BioMedical Materials and Engineering 19 147-53

[53] Watari F, Akasaka T, Ishikawa K, Matsuoka M, Hirata E, Terada N, Yokoyama A, Uo M, Itoh S, Yawaka Y, Suzuki M, Takashi N, Totsuka Y, Kitagawa Y, Abe S, Rosca I D, Kuboki Y and Bando Y 2010 Various nanotube scaffolds for cell proliferation Multiscale, Multifunctional and Functionally Graded Materials 631-632 181-6

[54] Zhang M, Fang S L, Zakhidov A A, Lee S B, Aliev A E, Williams C D, Atkinson K R and Baughman R H 2005 Strong, transparent, multifunctional, carbon nanotube sheets Science 309 1215-9

[55] Rebollar E, Frischauf I, Olbrich M, Peterbauer T, Hering S, Preiner J, Hinterdorfer P, Romanin C and Heitz J 2008 Proliferation of aligned mammalian cells on lasernanostructured polystyrene Biomaterials 29 1796-806

[56] Zhu B S, Lu Q H, Yin J, Hu J and Wang Z G 2004 Effects of laser-modified polystyrene substrate on $\mathrm{CHO}$ cell growth and alignment Journal of Biomedical Materials Research Part B-Applied Biomaterials 70B 43-8

[57] Peterbauer T, Yakunin S, Siegel J, Hering S, Fahrner M, Romanin C and Heitz J 2011 Dynamics of Spreading and Alignment of Cells Cultured In Vitro on a Grooved Polymer Surface Journal of Nanomaterials 413079

[58] Lima M r D, Fang S, Lepr $\tilde{A}^{3}$ X, Lewis C, Ovalle-Robles R, Carretero-GonzÃ $\tilde{i}_{\text {lez J, }}$ Castillo-MartÃnez E, Kozlov M E, Oh J, Rawat N, Haines C S, Haque M H, Aare V, 
Stoughton S, Zakhidov A A and Baughman R H 2011 Biscrolling Nanotube Sheets and Functional Guests into Yarns Science 331 51-5

[59] Rasband, W.S., ImageJ, U. S. National Institutes of Health, Bethesda, Maryland, USA, http://rsb.info.nih.gov/ij/, 1997-2009

[60] Discher D E, Janmey P and Wang Y L 2005 Tissue cells feel and respond to the stiffness of their substrate Science 310 1139-43

[61] Estevez M, Fernandez-Ulibarri I, Martinez E, Egea G and Samitier J 2010 Changes in the internal organization of the cell by microstructured substrates Soft Matter 6 582-90 


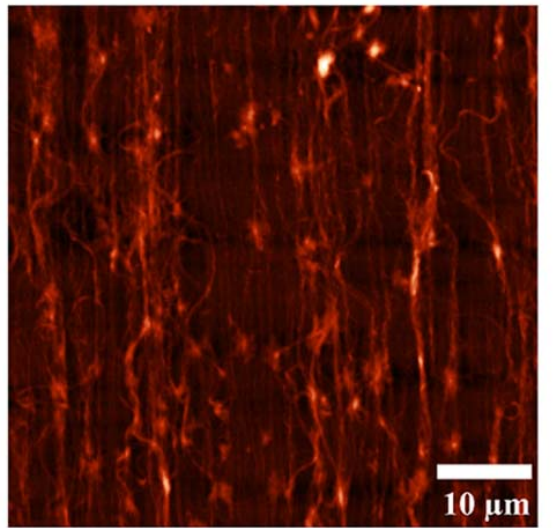

(A)

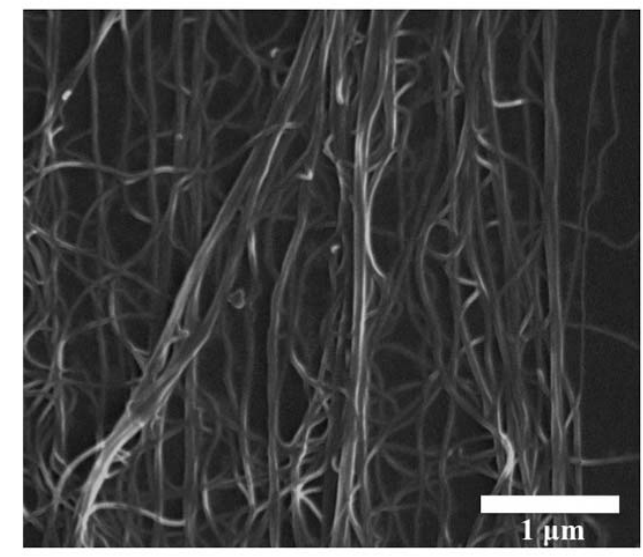

(C)

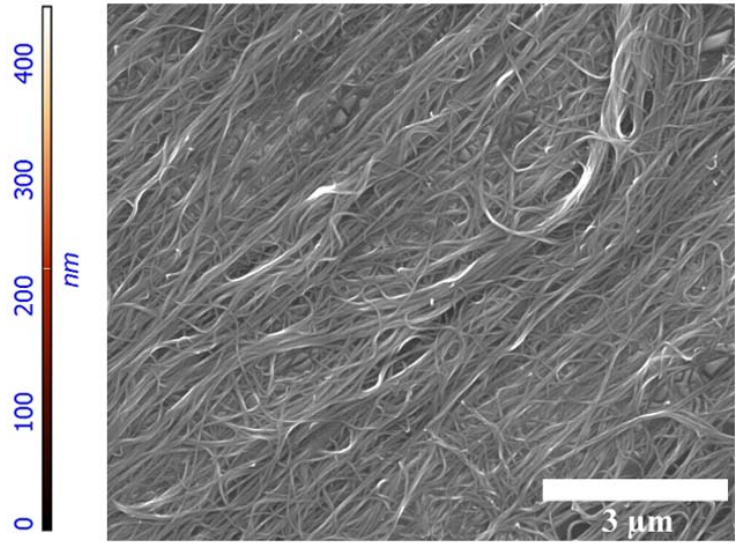

(B)

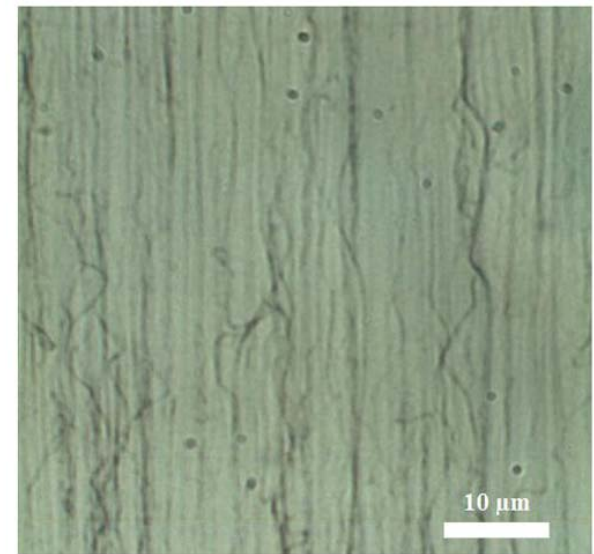

(D)

Figure 1. The topography of the aligned MWNT sheets. (A) and (B) are AFM height, and SEM images, respectively, of our sheets on glass. The cells were grown on these MWNT-on-glass substrates. (C) is a higher resolution image of an MWNT sheet on silicon. (D) is an optical microscopy image at 25x magnification of a MWNT sheet on glass In (A), (C), and (D) the MWNT bundles are vertical whereas in (B) they are angled at approximately $45^{\circ}$ to the vertical. 

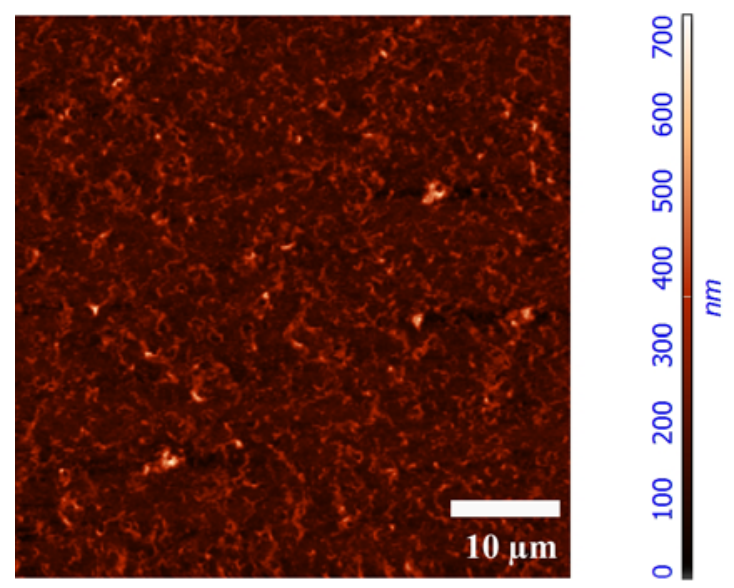

(A)

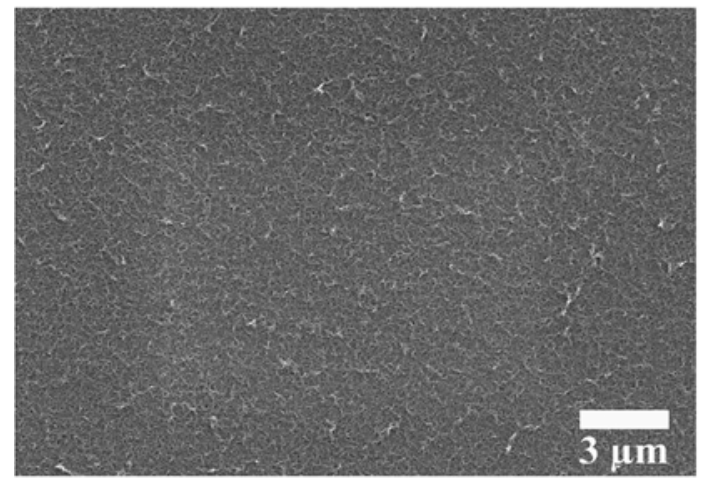

(B)

Figure 2. The topography of our isotropic buckypaper. (A) is an AFM height image, and (B) is an SEM image. 


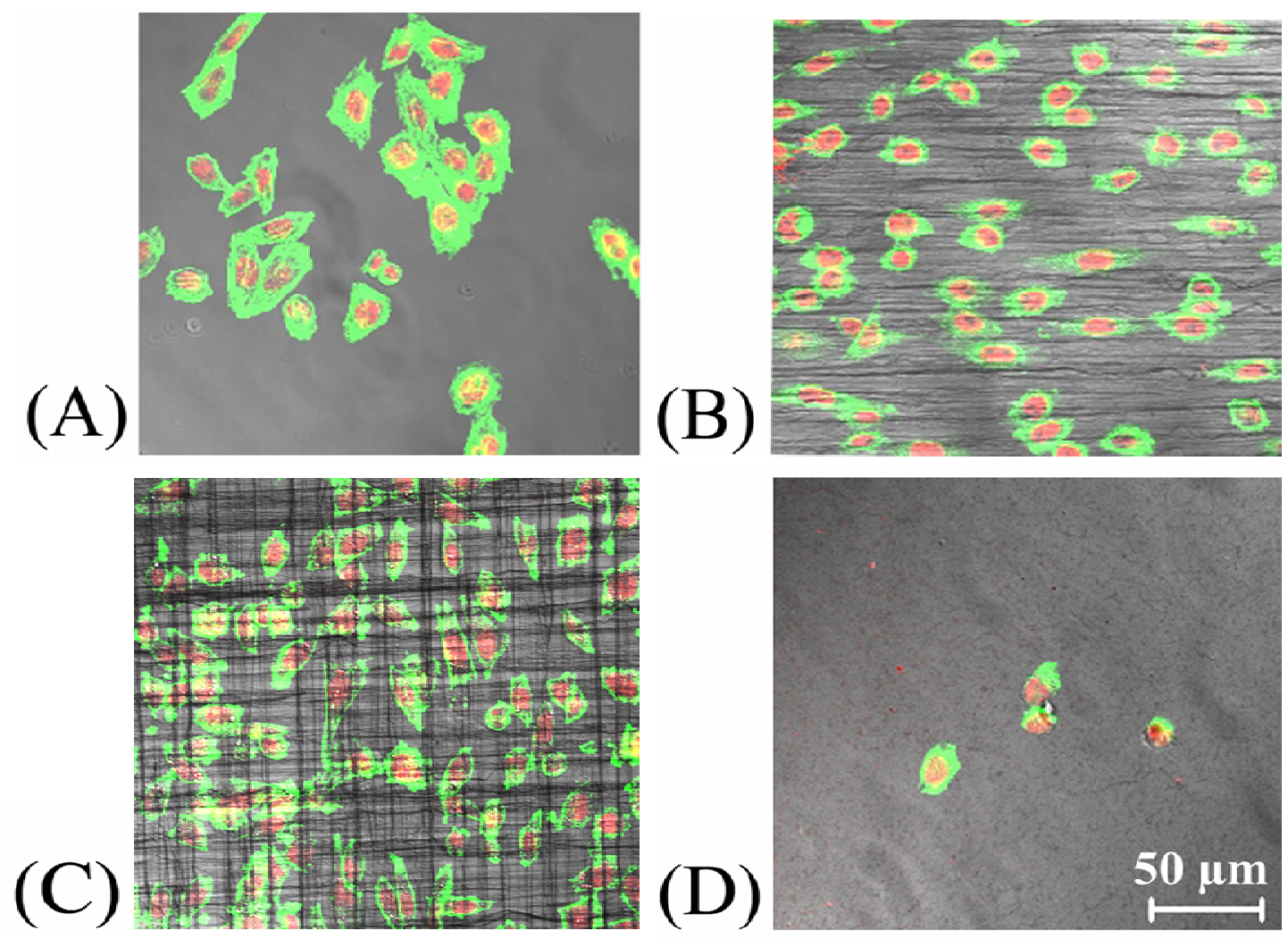

Figure 3. Confocal microscopy images of $\mathrm{CHO}$ cells on our substrates, and on control substrates (glass coverslips). The images are merged images of actin staining (green), nucleus staining (red), and Nomarski (DIC) images. In (A) the substrate is a glass coverslip, in (B) an anisotropic aligned MWNT substrate, in (C) it is two layers of aligned MWNT sheets, one on top of another, and in (D) it is isotropic buckypaper. In (B) the MWNTs are aligned horizontally and note that the cells align along these nanotube bundles. In (C) the top layer of MWNTs is horizontal and the bottom layer is vertical. All four images are at the same magnification. The scale bar is $50 \mu \mathrm{m}$. The images are taken after $20 \mathrm{hrs}$ on the substrates. 


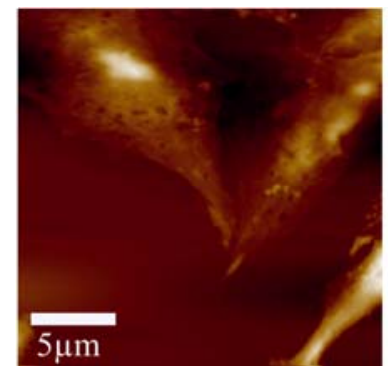

(A)

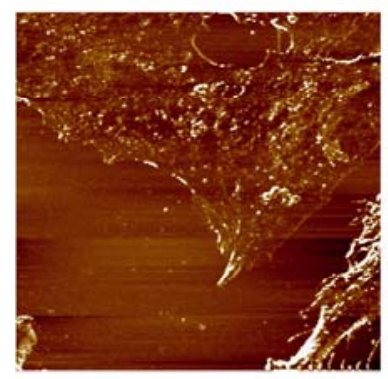

(B)

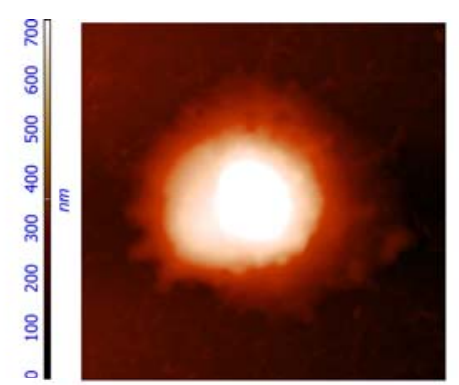

(C)

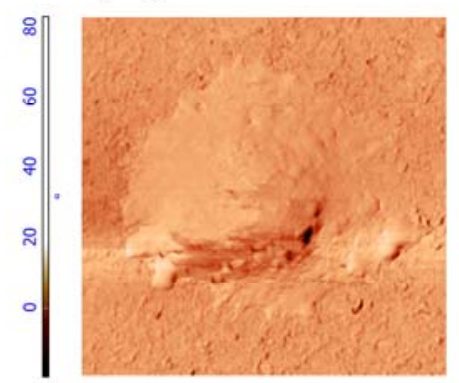

(D)

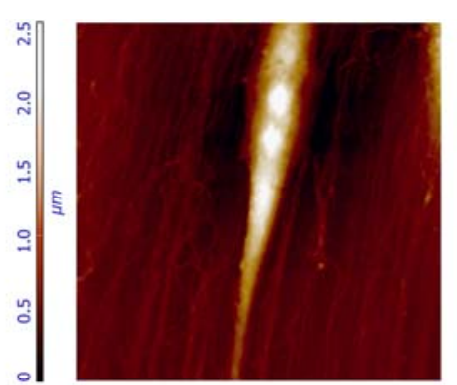

(E)

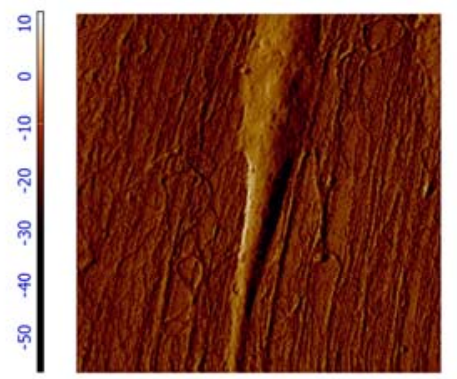

(F)
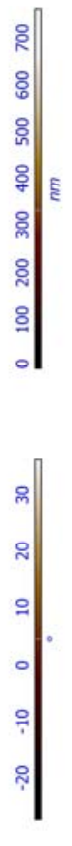

Figure 4. Tapping mode AFM topography (A, C and E) and phase (B, D and F) images of glutaraldehyde-fixed CHO cells on three different substrates. (A) and (B) are topography and phase images, respectively, of cells on a glass coverslip. (C) and (D) are also topography and phase images of the same area but here the substrate is isotropic buckypaper. (E) and (F) show a cell on our aligned MWNT substrate. In all cases the scan area is $20 \mu \mathrm{m} \times 20 \mu \mathrm{m}$. In (E) and (F) the MWNTs are visible and are aligned at a small angle clockwise of vertical. Note the elongated shape of the cell on the aligned MWNT bundles as compared to on the isotropic substrates. Also the cell in (E) and (F) is clearly aligned with the MWNT bundles. Bright areas in the height image are found to be the cell nucleus in our confocal microscopy images. The images were taken after $20 \mathrm{hrs}$ on the substrates. 


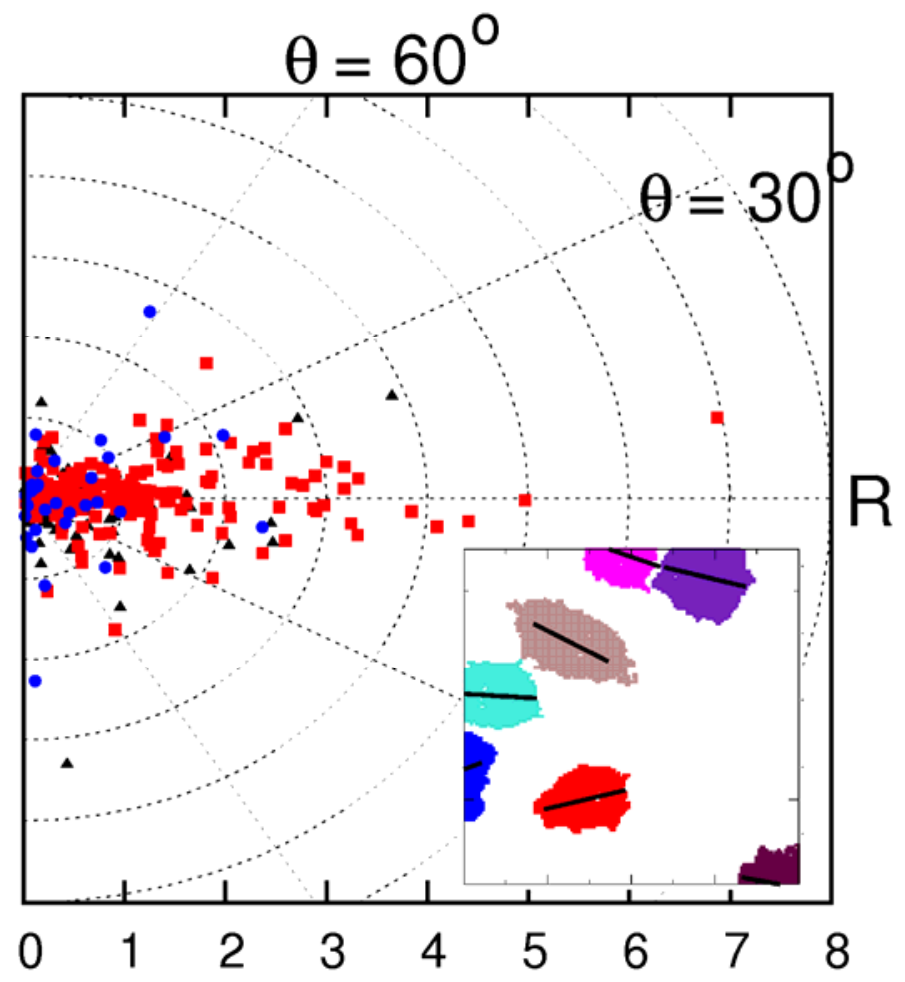

Figure 5. A polar plot for the elongation and orientation of cells or clusters of cells on our aligned MWNT substrates (red squares), on crossed MWNT sheets (black triangles) and on glass coverslips (blue circles). In this polar plot the distance from the origin, $\mathrm{R}$, is the elongation minus 1 and the angle to the horizontal, $\theta$, is the angle the long axis of the cell makes with the $x$-axis, which is the axis along which the MWNT bundles are aligned (the horizontal in Fig. 3(B)). The elongation is the ratio of the length of the long axis to length along an axis perpendicular to that axis. The numbers of cells or cell clusters are 191 for the aligned substrates, 95 for the crossed MWNT substrates and 31 on the control glass coverslips. The inset is an area of $40 \mu \mathrm{m} \times 40 \mu \mathrm{m}$ from one of our confocal images of cells on an aligned substrate. The image has been thresholded to produce a binary image and then each cell is shown in a different colour. The long axis of each cell is shown by a black line, which for convenience we have chosen to be $10 \mu \mathrm{m}$ long. 

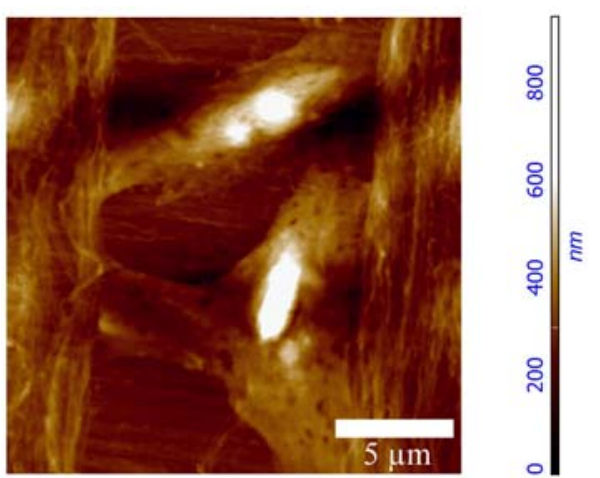

\section{(A)}
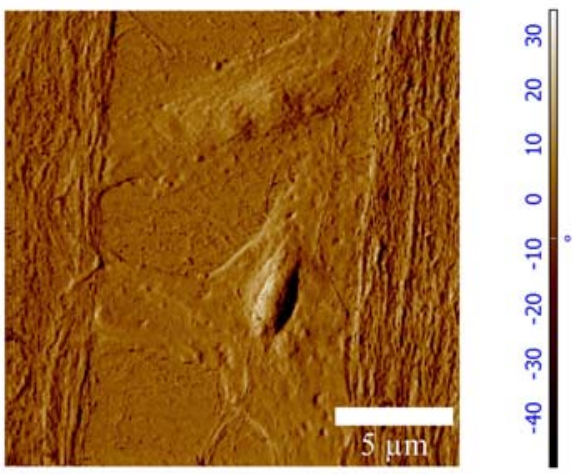

(B)

Figure 6. AFM height (A) and phase (B) images showing $\mathrm{CHO}$ cells bridging the gap between two parts of a substrate covered with vertically aligned MWNT bundles. Although not easily visible in the images, underneath the vertically aligned MWNT bundles and in the gap between them are MWNT bundles oriented horizontally. The gap between the vertical bundles is approximately $10 \mu \mathrm{m}$ across. Two cells are visible in the scan area of $18 \mu \mathrm{m} \times 18 \mu \mathrm{m}$ and both of them are growing in the gap, i.e., on a single sheet of horizontally oriented MWNT bundles, and both are stretching out to adhere to the MWNT bundles on the sides of this gap. The images are obtained from tapping mode AFM topography of glutaraldehyde-fixed $\mathrm{CHO}$ cells. The images were taken after $20 \mathrm{hrs}$ incubation on the substrates. 

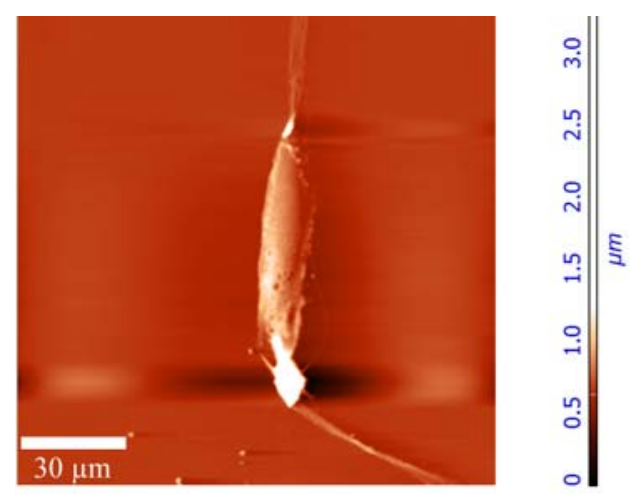

\section{(A)}
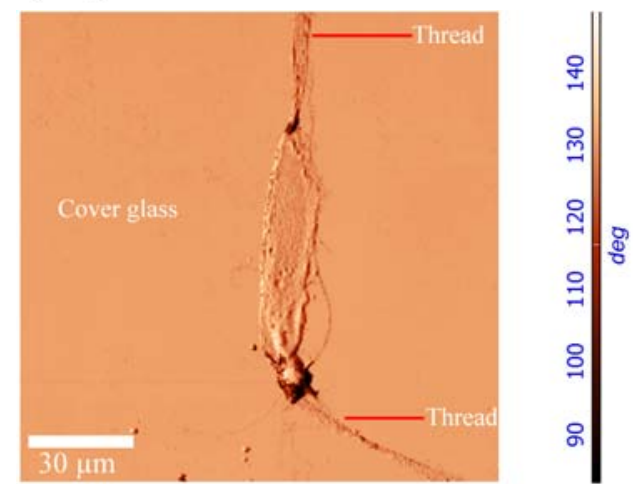

\section{(B)}

Figure 7. The cell morphology of CHO cells on a thread of MWNTs. The thread was obtained from one of our aligned sheets. (A) AFM height image (tapping mode). (B) AFM phase image of the same area. The thread is continuous, and so runs underneath the cell. Except where it is underneath the cell it is clearly visible in the phase image. The images were taken after $20 \mathrm{hrs}$ incubation on the substrate. 


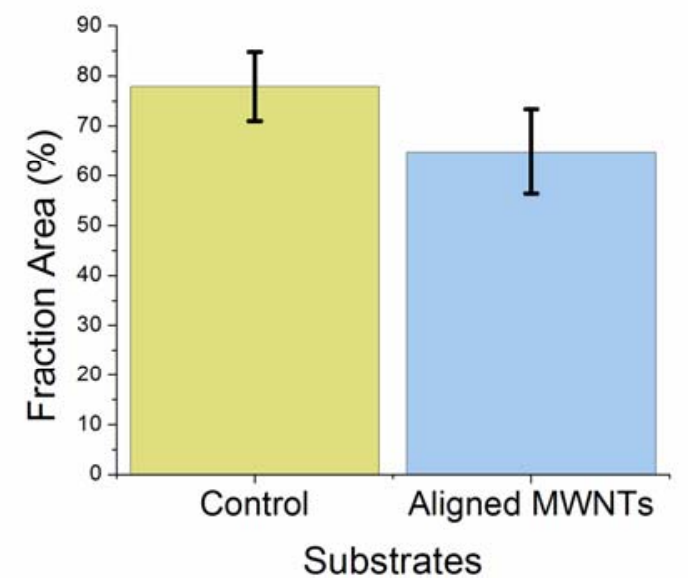

Figure 8. Graph showing column plot for the percentage of the substrate covered by CHO cells after 43 hours. 